\title{
Belphégor
}

\section{Éléonore Reverzy. Portrait de l'artiste en fille de joie. La littérature publique}

Sándor Kálai

\section{(2) OpenEdition}

1 Journals

\section{Édition électronique}

URL : https://journals.openedition.org/belphegor/4019

DOI : 10.4000/belphegor.4019

ISSN : 1499-7185

Éditeur

LPCM

\section{Référence électronique}

Sándor Kálai, «Éléonore Reverzy. Portrait de l'artiste en fille de joie. La littérature publique », Belphégor [En ligne], 19-1 | 2021, mis en ligne le 26 juin 2021, consulté le 06 juillet 2021. URL : http:// journals.openedition.org/belphegor/4019; DOI : https://doi.org/10.4000/belphegor.4019

Ce document a été généré automatiquement le 6 juillet 2021

\section{(c) (i) (9)}

Belphégor est mis à disposition selon les termes de la Licence Creative Commons Attribution - Pas d'Utilisation Commerciale - Pas de Modification 4.0 International. 


\title{
Éléonore Reverzy. Portrait de l'artiste en fille de joie. La littérature publique
}

\author{
Sándor Kálai
}

\section{RÉFÉRENCE}

Éléonore Reverzy : Portrait de l'artiste en fille de joie. La littérature publique. Paris, CNRS Éditions, 2016.

1 Éléonore Reverzy, spécialiste de la littérature française du $19^{\mathrm{e}}$ siècle, dont l'avantdernier livre, La chair de l'idée (Genève, 2007), est consacré à la poétique de l'allégorie dans l'œuvre de Zola, s'intéresse, dans le présent ouvrage, publié en 2016, à la problématique de ce qu'elle appelle «la littérature publique», c'est-à-dire à la littérature qui circule dans le contexte de la modernité (mass)médiatique. L'idée principale est que, dans ce contexte nouveau, l'écrivain et le journaliste doivent se vendre, vendre leurs produits, donc en quelque sorte ils se prostituent: c'est cette métaphore, celle de la prostituée - et la batterie discursive qui se déploie autour de ce noyau à partir de 1830 -, dont l'analyse organise les chapitres de l'ouvrage.

2 La prostituée, un type de personnage romanesque récurrent du siècle, peut être considérée, par la mobilité particulière qui la caractérise, comme un relais du regard réaliste. En tant que métaphore elle renvoie, entre autres, à la nouvelle condition de l'écrivain, qui peut de moins en moins se concevoir comme un mage. Éléonore Reverzy passe au crible, au fil des chapitres, une impressionnante quantité de textes (à la fois critiques, journalistiques ou fictionnels) et ses analyses se situent à l'intersection de l'histoire littéraire, de la sociologie de la littérature et de l'histoire culturelle. Les chapitres peuvent être lus séparément, mais l'ensemble de l'ouvrage est organisé d'un point de vue chronologique: depuis ce qu'Eléonore Reverzy identifie comme un moment charnière, autour de 1830, l'ouvrage suit son fil jusqu'à la littérature de la fin du $19^{e}$ siècle. L'analyse des modulations de la métaphore nécessite une attention particulière au caractère éminemment genré de la problématique. 
3 Les analyses d'Éléonore Reverzy trouvent leur place dans les récentes tendances de l'histoire littéraire française, qui, dans un dialogue fécond avec l'histoire culturelle, aborde la littérature comme un système de communication, accordant une attention particulière au contexte médiatique. Entre autres, les analyses d'Alain Vaillant mettent l'accent sur un changement de paradigme, survenu entre 1820 et 1850, dans l'espace européen : le paradigme argumentatif est progressivement remplacé par le paradigme narratif, l'écrit sert désormais à représenter le réel et l'écrivain comme médiateur se trouve pris entre les lecteurs et le réel. Le nouveau paradigme se déploie avant tout dans la presse, premier média de masse, à cause de ses caractéristiques: elle est médiatique, quotidienne et collective. Dans le sillage de cette tradition, Éléonore Reverzy passe en revue dans son introduction les caractéristiques de l'ère médiatique (déstabilisation des certitudes - que ce soit celles du public, des auteurs ou des genres; multiplication des supports ; professionnalisation ; salaire) et met en avant deux dates : 1830, surgissement des nouvelles lois du commerce de l'immatériel, et 1870, moment important du développement de la culture de masse.

4 Le premier chapitre est consacré au discours journalistique : l'auteure du livre passe en revue les articles du Journal des débats, du Figaro, de La Presse, du Constitutionnel et du Siècle publiés entre 1828 et 1848 . L'analyse des modulations de la métaphore permet de comprendre que la pratique littéraire et journalistique de l'époque signifie, pour ses acteurs, une perte de "virilité»: l'objet périodique est un immense champ des possibles, mais il représente aussi une menace.

5 Le chapitre suivant passe en revue les œuvres fondatrices : les romans de Balzac, de Sue et de Dumas fils enrichissent et complètent la métaphore. Il s'agit ici d'analyser l'importance de ces figures de femmes dans l'économie des récits, ou dans le panorama qu'un roman (tout particulièrement les Illusions perdues, une des œuvres capitales) peut donner sur le monde artistique ou dans le discours d'escorte (la préface des Chouans qui s'intègre dans le paradigme de l'exposition). Dans le cas des Mystères de Paris, la figure de la prostituée peut être considérée comme l'allégorie d'un texte qui s'inscrit dans le régime médiatique, tandis que l'histoire de Marguerite Gautier renvoie aussi à celle de son auteur.

6 A travers l'analyse des œuvres des Goncourt ou de Barbey d'Aurevilly le chapitre consacré à la littérature Barnum (publicité, annonce) passe en revue les poétiques de la réclame (par exemple les effets de surcharge destinés à séduire les lecteurs) et attire l'attention sur l'esthétique pédiculaire: le récit consacré aux petits faits envahit le journal (chronique, fait divers) et contamine les genres littéraires. C'est ici qu'on peut identifier un des ressorts de la sérialité: le feuilleton implique morcellement et publication en série.

7 Le quatrième chapitre du livre est consacré à ce qui est formulé dans le titre : à l'artiste comme prostituée. La fille qui peut aussi être associée à la sainteté (et à l'ascèse) permet de garder à la littérature sa grandeur (selon le paradigme flaubertien), tandis que dans la deuxième moitié du siècle, dans un autre contexte, l'artiste s'approprie la métaphore et intériorise ainsi, à la manière d'un Zola, les nouvelles conditions historiques de création (presse à un sou, extension du marché éditorial, impossibilité d'échapper à la culture de masse naissante).

8 Le cinquième chapitre, à travers l'analyse de L'Éducation sentimentale ou de Nana, cherche à éclairer le fonctionnement du commerce de l'immatériel, le marché de l'art. 
L'économie de la prostitution, qui interroge la logique du capitalisme, permet aussi de parler de l'art.

9 La prostituée peut aussi être considérée comme une figure qui peut révéler la vérité c'est à cette problématique que le chapitre suivant s'intéresse. L'exposition romanesque du corps s'inscrit dans le paradigme indiciaire, et tend vers une vérité que les récits veulent atteindre. Le thème du modèle dénudé, objet de tableaux exposés, permet de comprendre que les tableaux, tableaux vivants, photographies, sculptures figurant une femme nue véhiculent des représentations, souvent mythologiques, condensant une signification volontiers politique.

10 En tant que pendant ou miroir du deuxième chapitre, le septième est consacré aux romans de filles qui, selon le projet réaliste et naturaliste, parlent, à travers la figure de la prostituée, de l'argent et du sexe. L'écriture taxinomique qui les caractérise permet d'éclairer l'instabilité de la société démocratique. Une partie du chapitre est consacrée à la représentation romanesque et picturale/photographique de la fille lectrice : tandis que la fille qui est absorbée par sa lecture (Nana ou Élisa) place à distance le désir masculin ; celle qui regarde le spectateur l'invite à la rejoindre.

11 Le dernier chapitre de l'ouvrage aborde les lieux (boulevard, rue, vitrines, maisons) qui déterminent les actualisations narratives et les dispositifs visuels qui peuvent en résulter. L'analyse du lancement de Nana de Zola attire l'attention sur une des caractéristiques des nouvelles conditions, celle de la spectacularisation médiatique.

Ce rapide passage en revue des chapitres (qui ne permet que très imparfaitement de restituer les richesses du livre) témoigne de la réussite de l'entreprise : la métaphore persiste à travers le siècle et l'analyse fine d'Éléonore Reverzy passe en revue ses déclinaisons tout en tenant compte du changement du contexte qui survient entre 1830 et 1880. La prostituée permet l'allégorisation du nouveau système médiatique ou celle de ses différents acteurs, mais elle peut aussi être considérée comme un puissant «moteur » de la fiction, en lui conférant une dimension politique ou en l'inscrivant dans une perspective métapoétique, mais, avant tout, elle est une figure de la jouissance, et ce à plusieurs niveaux, elle "représenterait ainsi, dans un modèle économique bourgeois et thésauriseur, la part de folie, la dépense à fonds perdu... » (307).

13 Le livre d'Éléonore Reverzy est important à cause de l'analyse transversale qu'elle propose : ce qui l'intéresse ici, ce ne sont pas des œuvres ou des auteurs particuliers (même si ses auteurs de prédilection, Zola, les Goncourt ou autres, ont ici aussi une place de choix), mais une époque précise. Même si ce sont les ouvrages du $19^{\mathrm{e}}$ siècle qui constituent le pivot des analyses, les exemples sont aussi tirés de la littérature du $18^{\mathrm{e}}$ siècle (qui annonce donc les changements à venir). Cette archéologie de la modernité médiatique, à travers une optique particulière, permet d'éclairer un paradigme dans lequel nous continuons de vivre.

Les analyses s'intéressent surtout aux romans, genre éminent du nouveau régime culturel, mais l'auteure ne néglige ni la poésie, ni le théâtre (ces genres doivent s'inscrire eux aussi dans ce régime) et, comme nous l'avons noté, une attention particulière est accordée aux dispositifs visuels (représentés par les récits ou mis en parallèle avec eux).

15 L'ouvrage peut donc intéresser non seulement les dix-neuviémistes mais aussi ceux qui s'intéressent à la poétique journalistique ou à la littérature populaire. Comme l'analyse 
postule que le texte littéraire est inséparable des conditions de sa production, de sa circulation et de sa réception (support, processus éditorial, critique, public), le livre d'Éléonore Reverzy peut être lu avec d'autres qui s'intéressent à la publication (p. ex. Protocoles éditoriaux. Qu'est-ce que publier?, sous la direction d'Olivier Bomsel, Paris, A. Colin, 2013) ou à l'analyse du système médiatique dans lequel nous vivons et à son archéologie (p. ex. Yves Citton, Médiarchie, Paris, Seuil, 2017).

INDEX

Mots-clés : Zola, 19e siècle, Eleonore Reverzy

\section{AUTEUR}

SÁNDOR KÁLAI

Université de Debrecen 